\title{
Applying Multidimensional Geometry to Basic Data Centre Designs
}

\author{
Pedro Juan Roig ${ }^{1,2, *}$, Salvador Alcaraz ${ }^{1}$, Katja Gilly ${ }^{1}$, Carlos Juiz ${ }^{2}$ \\ ${ }^{1}$ Department of Computing Engineering, Miguel Hernández University, Spain, \\ Email:pedro.roig@graduado.umh.es,salcaraz@umh.es,katya@umh.es \\ ${ }^{2}$ Department of Mathematics and Computer Science, University of the Balearic Islands, Spain, \\ Email:cjuiz@uib.es \\ * Corresponding author
}

\begin{abstract}
Fog computing deployments are catching up by the day due to their advantages on latency and bandwidth compared to cloud implementations. Furthermore, the number of required hosts is usually far smaller, and so are the amount of switches needed to make the interconnections among them. In this paper, an approach based on multidimensional geometry is proposed for building up basic switching architectures for Data Centres, in a way that the most common convex regular $N$-polytopes are first introduced, where $N$ is treated in an incremental manner in order to reach a generic high-dimensional $N$, and in turn, those resulting shapes are associated with their corresponding switching topologies. This way, $N$-simplex is related to a full mesh pattern, $N$-orthoplex is linked to a quasi full mesh structure and $N$-hypercube is referred to as a certain type of partial mesh layout. In each of those three contexts, a model is to be built up, where switches are first identified, afterwards, their downlink ports leading to the end hosts are exposed, along with those host identifiers, as well as their uplink ports leading to their neighboring switches, and eventually, a pseudocode algorithm is designed, exposing how a packet coming in from any given port of a switch is to be forwarded through the proper outgoing port on its way to the destination host by using the appropriate arithmetic expressions in each particular case. Therefore, all those algorithmic models represent how their corresponding switches may work when dealing with user data traffic within a Data Centre, guiding it towards its destination.
\end{abstract}

Keywords: Data Centre, N-hypercube, N-orthoplex, Nsimplex, networking.

\section{INTRODUCTION}

Fog Computing paradigm is an extension of Cloud Computing, where the computing assets are moved to the edge of the network, thus improving some key performance indicators such as latency, jitter and bandwidth [1].

Fog deployments are ever growing thanks to the rise of new wireless communication standards allowing for faster transmissions, such as cellular $5 \mathrm{G}$ or Wi-Fi 6 [2].

Moreover, other technologies may take advantage of those improvements, such as Mobile Edge Computing or Mist Computing, facilitating a change of paradigm in online services and hyperconnectivity in the coming years [3].

However, it is to be noted that Fog environments are related to limited geographical areas compared to Cloud ones, as the former are localized in a certain area whereas the latter usually have a global scope. This implies that Fog domains may have far less users and workload, resulting in the need of much less hosts in their Data Centres [4].
Therefore, that leads to utilize a smaller amount of switches so as to link together all those hosts, thus allowing for the use of easier interconnection topologies among those switches [5].

Hence, Fog deployments permit the implementation of basic Data Centre designs, which may be modelled by means of elements of multidimensional geometry, specifically, by using different types of $\mathrm{N}$-polytopes.

The organization of the rest of the paper goes as follows: first, Section 2 introduces multidimensional geometry, next, Section 3 talks about platonic solids, Section 4 explains the Schläfli notation, afterwards, Section 5 exposes regular $N$ polytopes in Euclidean spaces, later on, Section 6 presents the $N$-simplex, in turn, Section 7 demonstrates the $N$-orthoplex, then, Section 8 exhibits the $N$-hypercube, and finally, Section 9 wraps it all up by drawing some final conclusions.

\section{MULTIDIMENSIONAL GEOMETRY}

It may be said that the concept of dimension in a mathematical object is related to the minimum number of necessary coordinates to specify any of its given points [6].

This way, an independent point may be considered to be an object with no dimensions $(O D)$, a line may be a 1-dimension object $(1 D)$, a plane may be a 2-dimension object $(2 D)$ and a space may be a 3 -dimension object $(3 D)$. As a matter of fact, our physical world is regarded as tridimensional, as any point within may be completely described by quoting its length, width and height.

Back in the ancient Greece, Euclid established the basis to define the classical geometry within the three-dimensional space. Also in that time, Pythagoras came up with his Pythagorean theorem, which permitted to measure distances within the Euclidean geometry, bringing about related concepts such as length, angle, orthogonality or parallelism, which are the core of geometry nowadays.

Since then, different types of Non-Euclidean geometries have been defined, either by applying some sort of constant curvature, such as in hyperbolic and elliptic geometries, or otherwise, non-constant curvature, such as in Riemannian geometry, even though the infinitesimal structure of any space always behaves as Euclidean.

Therefore, focusing on the Euclidean space, where $N=3$, it may be extended to any number of dimensions, where such a number may be any natural number $N$. This leads to consider multidimensional geometries to those Euclidean spaces with $N>3$, which may not correspond to our physical world, but just an extension of it. Additionally, it may also apply when $N<3$. 


\section{PLATONIC SOLIDS}

Back in the ancient Greece, Plato studied and classified the so called platonic solids [7]. Each one was a regular polyhedron, made up by the same type of regular polygons, hence, 3D convex regular structures being composed of just one type of $2 \mathrm{D}$ convex regular shape. Only 5 solids meet these specifications, shown in Fig. 1.
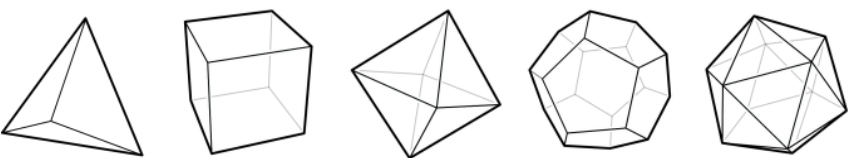

Fig. 1. Platonic solids

In this context, Plato associated them with the five classical basic elements (fire, earth, air, water and ether), such as the fire to the tetrahedron, the earth to the cube, the octahedron to the air, the icosahedron to the water, and eventually, the dodecahedron to the constellations in heaven, even though Aristotle later considered that heaven was made of ether.

Additionally, Table I exposes the numbers of faces, edges and nodes related to each of those platonic solids. Those data clearly reveal that there a two couple of shapes being dual of each other, such as the cube and the octahedron on the one hand, and the dodecahedron and the icosahedron on the other hand, as each pair of items has the same number of edges, and their number of faces and nodes are just switched around.

This basically means that the nodes of one item may be located right in the middle of the faces of its dual item, where the inner one is inscribed and the other one is circumscribed.

Besides, the tetrahedron is autodual, as the number of faces and nodes match, which makes that a tetrahedron may be inscribed or circumscribed to another tetrahedron.

TABLE I. K-FACETS ON THE PLATONIC SOLIDS

\begin{tabular}{|c|c|c|c|c|}
\hline $\begin{array}{c}\text { Name of the } \\
\text { Polyhedron }\end{array}$ & $\begin{array}{c}\text { Regular Polygons } \\
\text { as its faces }\end{array}$ & Faces & Edges & Nodes \\
\hline Tetrahedron & Triangle & 4 & 6 & 4 \\
\hline Cube & Square & 6 & 12 & 8 \\
\hline Octahedron & Triangle & 8 & 12 & 6 \\
\hline Dodecahedron & Pentagon & 12 & 30 & 20 \\
\hline Icosahedron & Triangle & 20 & 30 & 12 \\
\hline
\end{tabular}

In this sense, a $k$-facet may be defined as the component of dimension $k$ of a shape of dimension $N$, where $-1 \leq k \leq N$. Taking this into account, the most well-known instances regarding $k$-facets are the ones related to the physical world:

- $\quad k=-1$ is related to the empty set $(\varnothing)$, whose dimension is considered to be -1 and it represents empty space

- $\quad k=0$ is related to vertices (as its dimension is 0 )

- $\quad k=1$ is related to edges (as its dimension is 1 )

- $\quad k=2$ is related to faces (as its dimension is 2)

- $\quad k=3$ is related to cells (as its dimension is 3)

Furthermore, it is to be said that the number of $k$-facets for $k=-1$ and $k=N$ in a shape of dimension $N$ is always 1 according to set theory, as the former is assigned for consistency reasons and the latter denotes the shape itself.

As a side note, it is to be reminded that any shape of dimension $N$ is bounded by a number of shapes of dimension $N-1$, which means that a platonic solid $(3 D)$ is bounded by regular faces $(2 D)$, a face is surrounded by lines $(1 D)$, a line is limited by points $(O D)$, and a point has nothing around $(-1 D)$.

\section{SCHLÄFLI NOTATION}

Moving on to the XIX century, a swiss mathematician called Schläfli came up with a simple notation to describe any regular geometrical shape regardless its dimension, thus making possible its use for any regular $N$-polytope. This is known as Schläfli notation, or alternatively, Schläfli symbol.

Such a notation provides a summary of all the underlying $k$ facets of any regular polytope of dimension $N$, and it takes the form $\{p, q, r, \ldots, x, y, z\}$, having as many variables as $N-1$ [8].

Starting with this expression, by taking out variables on the right-hand side in an incremental way, it is revealed the different sorts of $k$-facets forming the overall shape. This way, $\{p, q, r, \ldots, x, y\}$ determines the type of its $(N-1)$-facets, whilst $\{p, q, r, \ldots, x\}$ denotes the kind of its $(N-2)$-facets, and all the way down to just $\{p\}$ giving the sort of its faces, also known as 2-facets. Therefore, it may be concluded that Schläfli symbols gives details about the different kinds of $k$-facets within any regular $N$-polytope.

Additionally, it is to be said that edges, being 1 -facets, would be represented by \{\} , because they are one-dimensional shapes and this notation grants them zero variables, which may seem obvious as edges only have one possible shape, regardless of its length. Furthermore, this notation may assign a negative number of variables to nodes, being 0 -facets, and to empty set, being the (-1)-facet, hence it does not apply to them.

On the other hand, a peak figure might be defined as the elements being incident on a given $k$-facet. Following with the Schläfli notation, by taking out variables on the left-hand side in an incremental way, it is revealed the different sorts of peak figures being incident on each kind of $k$-facet. This way, $\{q, r, \ldots, x, y, z\}$ stands for the vertex figure, that being an $(N-1)$ facet, $\{r, \ldots, x, y, z\}$ indicates the edge figure (the vertex figure of a vertex figure), that being an (N-2)-facet, and all the way down to just $\{z\}$ gives the $(N-3)$-peak figure, that being a 2 facet. Additionally, it is to be mentioned that \{\} would indicate the $(N-2)$-peak figure, that being a 1 -facet.

Alternatively, mixing both concepts together, such as $k$ facets and peak figures, it may be said that there is a number of $z(N-1)$-facets of type $\{p, q, \ldots, x, y\}$ around each $(N-3)$-facet.

\section{REGULAR $N$-POLYTOPES}

Starting with the $\mathbf{1 D}$ Euclidean space, \{\} is the only allowed symbol, which stands for a given edge. As stated above, there is only one type of shape, which in this case is the segment.

Moving on to the $2 D$ Euclidean space, $\{p\}$ indicates a convex regular polygon of $p$ edges, if $p$ is integer. For example, $\{3\}$ accounts for an equilateral triangle, $\{4\}$ for a square, $\{5\}$ for a regular pentagon, and so on, as stated in (1), where $p$ needs to be at least 3 so as to obtain a closed polygon.

$$
\forall p \in \mathbb{Z} / p \geq 3
$$

Furthermore, Fig. 2 shows the most common shapes related to convex regular polygons, along with its Schläfli symbol.

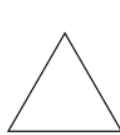

\{3\}

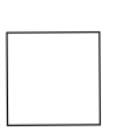

$\{4\}$

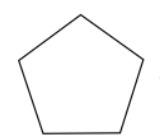

$\{5\}$
Fig. 2. Convex regular polygons.

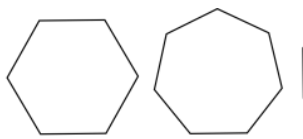

$\{7\}$

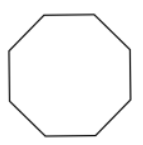

(8) 
Otherwise, it may seem clear that any convex regular polygon (2-facet) may not have any peak figure, as its vertex figure would be \{\} , standing for a segment ( 1 -facet), thus meaning that there are only two edges meeting at each vertex.

It may also happen that $p$ is rational, taking the form of a fraction $\{p / m\}$, with $p$ and $m$ being integer, which indicates a star regular polygon, or non-convex regular polygon. In such cases, the numerator $p$ states for the number of vertices of the star and the denominator $m$ does it for vertex interval step to build up the star.

In this sense, two conditions need to be met, given in (2). It is to be said that a compound polygon appears if the first condition is fulfilled, but the second one is not. For instance, only one pentagonal star is available $\{5 / 2\}$, whether there are two heptagonal stars $\{7 / 2\}$ or $\{7 / 3\}$, whilst $\{6 / 2\}$ makes it for a compound polygon, but not for a star regular one.

$$
\left\{\begin{array}{c}
\forall p, m \in \mathbb{Z} / \frac{p}{m}>2 \\
p, m \text { coprime }
\end{array}\right.
$$

Moreover, if the first condition of a star regular polygon is taken to the limit, such as shown in (3), then a straight line segment is obtained, also known as degenerate star polygon, which may be seen as a regular linear tiling of a $1 D$ space.

$$
\forall p, m \in \mathbb{Z} / \frac{p}{m}=2
$$

Moving ahead to $3 D$ Euclidean space, $\{p, q\}$ may indicate a convex regular polyhedron, where $\{p\}$ states the kind of convex regular polygons forming its faces and $\{q\}$ does it for the number of faces (2-facets) surrounding each of its vertices ( 0 -facets), also known as the vertex figure, whilst the edge figure is \{\} , meaning that there are just 2 faces meeting at every edge ( 1 -facets). Anyway, this kind of shape has to meet both conditions set in (4).

$$
\left\{\begin{array}{c}
\frac{1}{p}+\frac{1}{q}>\frac{1}{2}, \quad \forall p, q \in \mathbb{Z} / p, q \geq 3 \\
\{p\},\{q\} \subset \text { regular polygons }
\end{array}\right.
$$

The shapes meeting this criterion are also known as platonic solids and they have already been described above. However, Table II summarizes the Schläfli symbol for the five of them meeting the aforesaid expression.

TABLE II. PLATONIC SOLIDS - SCHLÄFLI NOTATION

\begin{tabular}{|c|c|c|c|c|}
\hline $\begin{array}{c}\text { Name of the } \\
\text { Polyhedron }\end{array}$ & $\begin{array}{c}\text { Regular Polygons } \\
\text { as its faces }\end{array}$ & $\{\boldsymbol{p}\}$ & $\{\boldsymbol{q}\}$ & $\{\boldsymbol{p}, \boldsymbol{q}\}$ \\
\hline Tetrahedron & Triangle & $\{3\}$ & $\{3\}$ & $\{3,3\}$ \\
\hline Cube & Square & $\{4\}$ & $\{3\}$ & $\{4,3\}$ \\
\hline Octahedron & Triangle & $\{3\}$ & $\{4\}$ & $\{3,4\}$ \\
\hline Dodecahedron & Pentagon & $\{5\}$ & $\{3\}$ & $\{5,3\}$ \\
\hline Icosahedron & Triangle & $\{3\}$ & $\{5\}$ & $\{3,5\}$ \\
\hline
\end{tabular}

Furthermore, star regular polyhedra, known as non-convex regular polyhedra or Kepler-Poinsot polyhedra, are made if either $p$ or $q$ are rational, specifically $5 / 2$, and the other value is either 3 or 5 . This accounts for a combination of a pentagonal star, with a dodecahedron or an icosahedron. Basically, there are just four possible combinations, such as just $\{5 / 2,3\},\{3,5 / 2\},\{5 / 2,5\},\{5,5 / 2\}$, which are two pairs of dual items. This way, (5) is met and a finite figure is attained.

$$
\frac{1}{p}+\frac{1}{q}>\frac{1}{2}, \quad\left\{\begin{array}{c}
\forall p \in \mathbb{Z} / p=3,5, q=5 / 2 \\
O R \\
\forall p \in \mathbb{Z} / p=5 / 2, q=3,5
\end{array}\right.
$$

Besides, bringing the condition for regular polyhedra to the limit, such as shown in (6), a complete regular plane tiling of a $2 D$ space may be achieved. This type of regular tessellation is the most cited one and only applies to the triangular tiling: $\{3,6\}$, the square tiling: $\{4,4\}$ and the hexagonal tiling: $\{6,3\}$.

$$
\frac{1}{p}+\frac{1}{q}=\frac{1}{2}, \quad \forall p, q \in \mathbb{Z} / p, q \geq 3
$$

Those three regular tessellation patterns may also be seen as periodic, because they show both translational and rotational symmetry. Another interesting type of tessellation is the aperiodic, which shows rotational symmetry but not translational one. However, this is not a regular tiling, as it needs more than one type of shape, making that condition (6) is not fulfilled.

Moving forward to four dimensions, also known as $\mathbf{4 D}$ Euclidean space, $\{p, q, r\}$ may indicate a convex regular polychoron (4-polytope) where both conditions in (7) need to be met. It is to be said that those shapes may have $r$ regular polyhedral cells $\{p, q\}$ surrounding each edge, whilst its cells are made of $\{p\}$ faces. Otherwise, each vertex figure is given by $\{q, r\}$, the edge figure is $\{r\}$ and the face figure is \{\} .

$$
\begin{gathered}
\operatorname{sen}\left(\frac{\pi}{p}\right)+\operatorname{sen}\left(\frac{\pi}{r}\right)-\cos \left(\frac{\pi}{q}\right)>0,\left\{\begin{array}{l}
\forall p, q, r \in \mathbb{Z} \\
p, q, r \geq 3
\end{array}\right. \\
\{p, q\},\{q, r\} \subset \text { regular polyhedra }
\end{gathered}
$$

The shapes meeting both conditions are just six, which are summarized in Table III.

TABLE III. CONVEX REGULAR 4-POLYTOPES - SCHLÄFLI NOTATION

\begin{tabular}{|c|c|c|c|c|c|}
\hline $\begin{array}{c}\text { Name of the } \\
\text { Polychoron }\end{array}$ & $\begin{array}{c}\text { Alternative } \\
\text { name }\end{array}$ & $\{\boldsymbol{p}\}$ & $\{\boldsymbol{q}\}$ & $\{\mathbf{r}\}$ & $\{\boldsymbol{p}, \boldsymbol{q}, \boldsymbol{r}\}$ \\
\hline Pentachoron & 4-simplex & $\{3\}$ & $\{3\}$ & $\{3\}$ & $\{3,3,3\}$ \\
\hline Tesseract & 4-hypercube & $\{4\}$ & $\{3\}$ & $\{3\}$ & $\{4,3,3\}$ \\
\hline Hexadecachoron & 4-orthoplex & $\{3\}$ & $\{3\}$ & $\{4\}$ & $\{3,3,4\}$ \\
\hline Icositetrachoron & 24-cell & $\{3\}$ & $\{4\}$ & $\{3\}$ & $\{3,4,3\}$ \\
\hline Hecatonicosachoron & 120 -cell & $\{5\}$ & $\{3\}$ & $\{3\}$ & $\{5,3,3\}$ \\
\hline Hexacosichoron & 600 -cell & $\{3\}$ & $\{3\}$ & $\{5\}$ & $\{3,3,5\}$ \\
\hline
\end{tabular}

Additionally, star regular polychora, also known as Schläfli-Hess polychora, are available only in combinations with the $5 / 2$ value involved in with star regular polyhedral, along with values 3 and 5 . There are only 10 combinations available, such as $\{3,5,5 / 2\},\{5,5 / 2,5\},\{5,3,5 / 2\},\{5 / 2,5,3\}$, $\{5,5 / 2,3\}, \quad\{5 / 2,3,5\}, \quad\{5 / 2,5,5 / 2\}, \quad\{3,5 / 2,5\}, \quad\{3,3,5 / 2\}$, $\{5 / 2,3,3\}$. This way, (8) is met and a finite figure is attained.

$$
\left\{\begin{array}{c}
\operatorname{sen}\left(\frac{\pi}{p}\right)+\operatorname{sen}\left(\frac{\pi}{r}\right)>\cos \left(\frac{\pi}{q}\right),\left\{p, q, r \in 3,5, \frac{5}{2},\right. \\
\{p, q\},\{q, r\} \subset \text { regular polyhedra }
\end{array}\right.
$$

Moreover, taking the condition for regular polychora to the limit, such as shown in (9), a complete regular tiling of a $3 D$ space may be attained only with a regular cubic tessellation, given by $\{4,3,4\}$, where dihedral angle constraint is set to 0 . 


$$
\begin{gathered}
\operatorname{sen}\left(\frac{\pi}{p}\right)+\operatorname{sen}\left(\frac{\pi}{r}\right)-\cos \left(\frac{\pi}{q}\right)=0,\left\{\begin{array}{c}
\forall p, q, r \in \mathbb{Z} \\
p, q, r \geq 3
\end{array}\right. \\
\{p, q\},\{q, r\} \subset \text { regular polyhedra }
\end{gathered}
$$

Moving on to five dimensions, also known as $\mathbf{5 D}$ Euclidean space, it is to be noted that convex regular polytera may be defined by $\{p, q, r, s\}$, where both conditions given in (10) are fulfilled. It is to be mentioned that these shapes may have $s$ regular 4-polytopes of type $\{p, q, r\}$ surrounding each face, whereas its cells are $\{p, q\}$ and its faces are $\{p\}$. Otherwise, the vertex figure is given by $\{q, r, s\}$, the edge figure is $\{r, s\}$, the face figure is $\{p\}$ and the cell figure is \{\} .

$$
\left\{\begin{array}{c}
\frac{\cos ^{2}\left(\frac{\pi}{q}\right)}{\sin ^{2}\left(\frac{\pi}{p}\right)}+\frac{\cos ^{2}\left(\frac{\pi}{r}\right)}{\sin ^{2}\left(\frac{\pi}{s}\right)}<1,\left\{\begin{array}{l}
\forall p, q, r, s \in \mathbb{Z} \\
p, q, r, s \geq 3
\end{array}\right. \\
\{p, q, r\},\{q, r, s\} \subset \text { regular polychora }
\end{array}\right.
$$

There are only three combinations available, which are quoted in Table IV.

TABLE IV. CONVEX REGULAR 5-POLYTOPES - SCHLÄFLI NOTATION

\begin{tabular}{|c|c|c|c|c|c|c|}
\hline $\begin{array}{c}\text { Name of the } \\
\text { Polyhedron }\end{array}$ & $\begin{array}{c}\text { Alternative } \\
\text { name }\end{array}$ & $\{\boldsymbol{p}\}$ & $\{\boldsymbol{q}\}$ & $\{\mathbf{r}\}$ & $\{\mathbf{s}\}$ & $\{\boldsymbol{p}, \boldsymbol{q}, \boldsymbol{r}, \boldsymbol{s}\}$ \\
\hline Hexateron & 5-simplex & $\{3\}$ & $\{3\}$ & $\{3\}$ & $\{3\}$ & $\{3,3,3,3\}$ \\
\hline Penteract & 5-hypercube & $\{4\}$ & $\{3\}$ & $\{3\}$ & $\{3\}$ & $\{4,3,3,3\}$ \\
\hline Pentacross & 5-orthoplex & $\{3\}$ & $\{3\}$ & $\{3\}$ & $\{4\}$ & $\{3,3,3,4\}$ \\
\hline
\end{tabular}

Generalizing, in Euclidean spaces of dimension $N>4$, the Schläfli symbol for each $N$-polytope may be $\{p, q, \ldots, y, z\}$, formed by a number of $z$ regular $N$-1-polytopes of type $\{p, q, \ldots, y\}$ around each $(N-3)$-facet, where $\{q, \ldots, y, z\}$ accounts for the vertex figure. Furthermore, each of the $k$-facets may be recursively analyzed as exposed in the previous cases.

However, the only convex regular $N$-polytopes in dimensions $N>4$ are the following:

- $\quad$ the $N$-simplex, with Schläfli notation $\{3,3, \ldots, 3\}$

- $\quad$ the $N$-hypercube, with Schläfli notation $\{4,3, \ldots, 3\}$

- $\quad$ the $N$-orthoplex, with Schläfli notation $\{3,3, \ldots, 4\}$

Additionally, it is to be noted that $N$-simplex may be referred to as regular simplices, $N$-hypercube as measure polytopes and $N$-orthoplex as cross polytopes. By looking at the notation, a measure polytope may be seen as dual of a cross polytope of the same dimension, meaning they may interchange the $i$ dimensional elements of one of them for the $N-i$ dimensional elements of its dual one, starting by $i=0$ (nodes), $i=1$ (edges), and going all the way to $i=(N-1)$-facets.

It is also to be noted that the symbol in Schläfli notation for those $N$-polytopes is the same Schläfli symbol flipped around. This way, it may seem clear that $N$-hypercube $\{4,3, \ldots, 3\}$ and $N$-orthoplex $\{3,3, \ldots, 4\}$ are dual of each other, as their symbols are just turned around, whereas the $N$-simplex is autodual, as its symbol contains only $3 \mathrm{~s}\{3,3, \ldots, 3,3\}$, matching both ways.

Otherwise, there are no star regular polytera, as conditions are not met, which may be generalized to say that there is no star regular $N$-polytope for any dimension $N>4$.

Furthermore, moving the condition for regular 5-polytopes to the limit, as seen in (11), a complete regular 4D-space tiling is achieved by three regular 4-polytope tessellations, whose symbols are $\{4,3,3,4\},\{3,3,4,3\}$ and $\{3,4,3,3\}$.

$$
\left\{\begin{array}{c}
\frac{\cos ^{2}\left(\frac{\pi}{q}\right)}{\sin ^{2}\left(\frac{\pi}{p}\right)}+\frac{\cos ^{2}\left(\frac{\pi}{r}\right)}{\sin ^{2}\left(\frac{\pi}{s}\right)}=1,\left\{\begin{array}{l}
\forall p, q, r, s \in \mathbb{Z} \\
p, q, r, s \geq 3
\end{array}\right. \\
\{p, q, r\},\{q, r, s\} \subset \text { regular polychora }
\end{array}\right.
$$

In summary, for higher Euclidean spaces, the only convex regular $N$-polytopes are the $N$-simplex, the $N$-hypercube and the $N$-orthoplex. Therefore, those three shapes are going to be reviewed for a generic dimension $N$ in order to associate them with basic Data Centre designs.

\section{N-SIMPLEX: FULL MESH}

Focusing on Data Centre designs, the most interesting feature in the $N$-simplex shape is that all its nodes are linked together no matter the value of dimension $N$, as seen in Fig. 3.

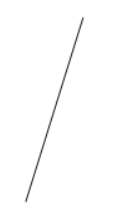

1-simplex

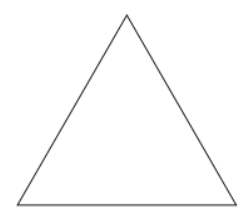

2-simplex

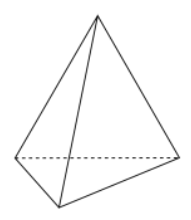

3-simplex

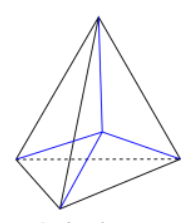

4-simplex
Fig. 3. $N$-simplex for some values of dimension $N$.

This topology perfectly fits into the full mesh topology [9], hence, its characteristics may be applied to obtain a model for it. Hence, the geometric features of an $\mathrm{N}$-simplex are going to be presented, such as nodes, edges, faces, ...

As a side note, it is to be mentioned that the Euler characteristic may be used so as to check out the results obtained next. Euler characteristic $(\chi)$ works for any geometrical shape as a topological invariant, in a way that it describes topological shapes. Focusing on convex regular $N$ polytopes, regardless its type or dimension, they may be seen as being homeomorphic to a topological $(N-1)$-sphere, so in this case, $\chi$ is given by (12).

$$
\chi=\sum_{i=0}^{N-1} N_{i}=N_{0}-N_{1}+N_{2}-N_{3} \ldots=1-(-1)^{N}
$$

Getting back to an $N$-simplex, the number of $k$-facets contained in such a shape of a given dimension $N$ may be achieved by applying expression (13).

$$
\left(\begin{array}{l}
N+1 \\
k+1
\end{array}\right)=\frac{(N+1) !}{(k+1) ! \cdot((N+1)-(k+1)) !}
$$

The same results obtained by means of the aforesaid combinatory expression, may also be attained by spotting the value present in the $(N+1)$ row and the $(k+1)$ column of the Pascal's triangle, shown in Fig. 4.

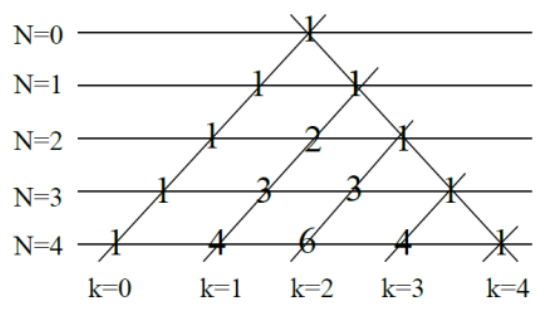

Fig. 4. Pascal's triangle for the first rows. 
The values for the $k$-facets related to the lower values of $N$ are shown in Table V, where the empty set are also include to fully appreciate the relationship with the Pascal's triangle.

TABLE V. AMOUNT OF K-FACETS PRESENT IN AN N-SIMPLEX

\begin{tabular}{|c|c|c|c|c|c|c|}
\hline & $\begin{array}{c}(\emptyset) \\
k=-1\end{array}$ & $\begin{array}{c}\text { Nodes } \\
k=0\end{array}$ & $\begin{array}{c}\text { Edges } \\
k=1\end{array}$ & $\begin{array}{c}\text { Faces } \\
\boldsymbol{k}=\mathbf{2}\end{array}$ & $\begin{array}{c}\text { Cells } \\
\boldsymbol{k}=3\end{array}$ & $\begin{array}{c}\text { 4-polyt. } \\
\boldsymbol{k}=\mathbf{4}\end{array}$ \\
\hline $\mathrm{N}$ & $\left(\begin{array}{c}N+1 \\
0\end{array}\right)$ & $\left(\begin{array}{c}N+1 \\
1\end{array}\right)$ & $\left(\begin{array}{c}N+1 \\
2\end{array}\right)$ & $\left(\begin{array}{c}N+1 \\
3\end{array}\right)$ & $\left(\begin{array}{c}N+1 \\
4\end{array}\right)$ & $\left(\begin{array}{c}N+1 \\
5\end{array}\right)$ \\
\hline-1 & 1 & N/A & N/A & N/A & N/A & N/A \\
\hline 0 & 1 & 1 & N/A & N/A & N/A & N/A \\
\hline 1 & 1 & 2 & 1 & N/A & N/A & N/A \\
\hline 2 & 1 & 3 & 3 & 1 & N/A & N/A \\
\hline 3 & 1 & 4 & 6 & 4 & 1 & N/A \\
\hline 4 & 1 & 5 & 10 & 10 & 5 & 1 \\
\hline
\end{tabular}

The most interesting features when dealing with Data Centre topologies will be nodes and edges, hence those are going to be the ones taken into consideration. Regarding the former, it is to be said that it follows the expression (14), whereas the latter does it to the expression (15). Hence, these expressions are equivalent to the ones cited above in Table V.

$$
\begin{gathered}
n^{\underline{\mathrm{o}}} \text { nodes }=N+1 \\
n^{\mathrm{o}} \text { edges }=N \cdot(N-1) / 2
\end{gathered}
$$

Thus, once the amount of nodes and edges have been found, a model is to be presented so as to apply it for the forwarding through the different switches of user data traffic among hosts being interconnected by a full mesh topology.

In this context, a nomenclature needs to be proposed for the hosts and switches making part of the full mesh topology. With respect to the hosts $h$, it is going to be set a given number $M$ connected to all switches, although a power of 2 might do, namely $M=2^{L}$. This way, the downlink ports of each switch go from 0 to $M-1$, whereas the hosts connected to each switch $i$ go from $i \cdot M$ all the way to $i \cdot(M+1)-1$. All of this is shown in Fig. 5. As per the behavior of the hosts, the role of them within this model is either to send a message through its unique port 0 , or otherwise, to receive a message through its only port 0 .

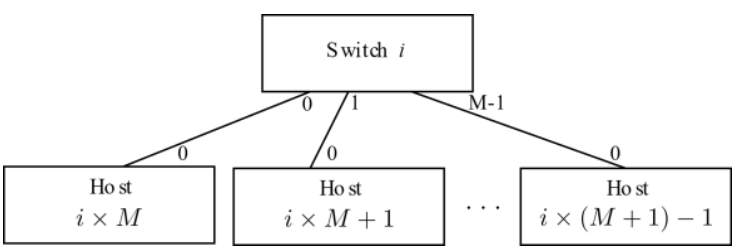

Fig. 5. Model for a generic switch $i$ with downlink ports connected to hosts.

With regards to the switches, there are going to be one located in each vertex of the $N$-polytope in order to achieve a full mesh topology, thus the total amount is given by $N+1$, as stated before for the number of nodes. Hence, this layout brings a total amount of $M \cdot(N+1)$ hosts in the whole topology, ranging from $h=0$, all the way to $h=M \cdot(N+1)-1$.

As per the nomenclature of those switches $i$, one of them is identified by 0 , and in turn, the rest will be identified by the dimension where they are standing with respect to the initial one, all the way to $N$, as exhibited in Fig. 6.

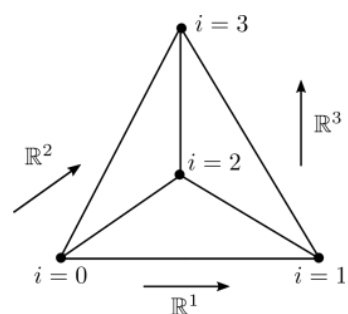

Fig. 6. Model the interconnected switches $i$ for 3-simplex.

Focusing on the links among switches, these will be located on each of the edges of the $N$-polytope in order to attain a full mesh topology, accounting its total amount to $N \cdot(N-1) / 2$, as stated before for the number of edges.

Getting down to the nomenclature of the uplink ports of each switch taking part on the interswitching links, the ports will go from $M$ to $M+N$, except for port $M+i$ in each switch $i$, as that port would connect to itself. This is shown in Fig. 7.

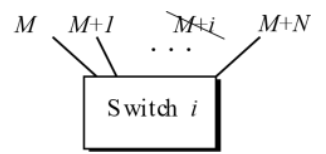

Fig. 7. Model for a generic switch $i$ for $N$-simplex with its uplink ports.

Eventually, it is possible to express the user data traffic flows by means of pseudocode, considering that $a$ and $b$ are source and destination hosts. It is to be noted that atomic actions send and receive need two arguments, such as the switch $i$ we are dealing with and the port where that action gets executed, being a generic port $p$ or otherwise.

With that all in mind, Algorithm 1 exposes the behavior of a generic switch $i$ regarding how incoming messages are forwarded on towards target host $b$.

Basically, it goes through all switches in order to check if any packet comes into any of its ports, and if so, it is verified if switch $i$ is the destination switch $\operatorname{int}(b / M)$, whose expression is due to the values exposed in Fig. 5. If that is the case, then the incoming packet is forwarded on to port $(b \bmod M)$, which leads directly to destination host, or otherwise, if switch $i$ is the source switch $\operatorname{int}(a / M)$, then the packet is forwarded on through all its uplink ports, thus getting redundancy, or else, the packet is sent out straight to the destination switch through port $M+\operatorname{int}(b / M)$, to avoid packets bouncing up and down.

Algorithm 1: Pseudocode for switches in full mesh topology $(N$-simplex $)$

01: While TRUE do

02: For $i$ in $0 \ldots N$ do

03: Receive (i, p)

04: If $i=\operatorname{int}(b / M)$ then

05: Send (i, $b \bmod M$ )

06: ElseIf $i=\operatorname{int}(a / M)$ then

07: For $q$ in $0 \ldots N$ do

08: If $q \neq i$ then

09: Send (i, $M+q$ )

10: End If

11: End For

12: Else

13: Send (i, $M+i n t(b / M)$ )

14: End If

15: End For

16: End While 


\section{VII. $\quad N$-ORTHOPLEX: QUASI FULL MESH}

Regarding Data Centre designs, the most interesting feature in the $\mathrm{N}$-orthoplex shape is that each node is linked together to the rest, except to its opposite one, no matter the value of dimension $N$, as shown in Fig. 8.

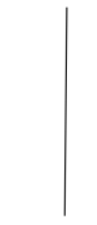

1-orthoplex

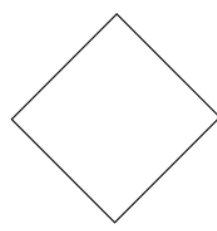

2-orthoplex

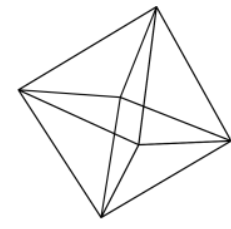

3-orthoplex

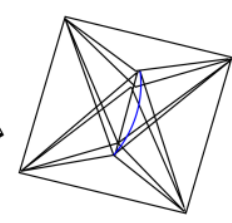

4-orthoplex
Fig. 8. $N$-orthoplex for some values of dimension $N$.

That topology fits into the quasi full mesh topology, where each node has a direct link to all the others, but not to its opposite one [10]. It results in each node having $2 \cdot(\mathrm{N}-1)$ neighbors and an opposite node being two hops away, with $2 \cdot(N-1)$ redundant paths to get there.

Hence, number of $k$-facets contained in an $N$-orthoplex of a given dimension $N$ may be calculated through expression (16).

$$
2^{k+1}\left(\begin{array}{c}
N \\
k+1
\end{array}\right)=2^{k+1} \cdot \frac{N !}{(k+1) ! \cdot(N-(k+1)) !}
$$

Applying the same reasoning than on the previous sections, Table VI presents the main features for dimension $N$.

TABLE VI. AMOUNT OF K-FACETS PRESENT IN AN N-ORTHOPLEX

\begin{tabular}{|c|c|c|c|c|c|c|}
\hline & $\begin{array}{c}(\varnothing) \\
k=-1\end{array}$ & $\begin{array}{c}\text { Nodes } \\
k=0\end{array}$ & $\begin{array}{c}\text { Edges } \\
\boldsymbol{k}=\mathbf{1}\end{array}$ & $\begin{array}{c}\text { Faces } \\
\boldsymbol{k}=\mathbf{2}\end{array}$ & $\begin{array}{c}\text { Cells } \\
\boldsymbol{k}=\mathbf{3}\end{array}$ & $\begin{array}{c}\text { 4-polyt. } \\
\boldsymbol{k}=\mathbf{4}\end{array}$ \\
\hline $\mathrm{N}$ & $2^{0}\left(\begin{array}{l}N \\
0\end{array}\right)$ & $2^{1}\left(\begin{array}{l}N \\
1\end{array}\right)$ & $2^{2}\left(\begin{array}{l}N \\
2\end{array}\right)$ & $2^{3}\left(\begin{array}{l}N \\
3\end{array}\right)$ & $2^{4}\left(\begin{array}{l}N \\
4\end{array}\right)$ & $2^{5}\left(\begin{array}{l}N \\
5\end{array}\right)$ \\
\hline-1 & 1 & N/A & N/A & N/A & N/A & N/A \\
\hline 0 & 1 & 1 & N/A & N/A & N/A & N/A \\
\hline 1 & 1 & 2 & 1 & N/A & N/A & N/A \\
\hline 2 & 1 & 4 & 4 & 1 & N/A & N/A \\
\hline 3 & 1 & 6 & 12 & 8 & 1 & N/A \\
\hline 4 & 1 & 8 & 24 & 32 & 16 & 1 \\
\hline
\end{tabular}

Otherwise, the main characteristics when dealing with Data Centre designs are the number of nodes (17) and edges (18), whose expressions are equivalents to those quoted in Table VI.

$$
\begin{gathered}
n^{\mathrm{o}} \text { nodes }=2 N \\
n^{\mathrm{o}} \text { edges }=2 N \cdot(N-1)
\end{gathered}
$$

Regarding the hosts connected to each switch $i$, the explanation given in the previous sections applies, such as in Fig. 5. However, the total amount of hosts depend on the number of switches, which is $2 N$, thus the overall number of hosts is given by $2 N \cdot M$, going from $h=0$ to $h=2 N \cdot M-1$.

As per the nomenclature of the switches $i$, which will be situated at each vertex of the $N$-orthoplex, one of them is identified by 0 , whereas its opposite node is $N$. Then, nodes from 1 to $N-1$ will be identified by the dimension where they are standing with respect to the initial one. Additionally, the rest of the nodes (from $N$ to $2 N-1$ ) will be allocated such that the opposite node of a node $i$ is generically identified by the expression $i \bmod 2 N$. This is all depicted in Fig. 9.

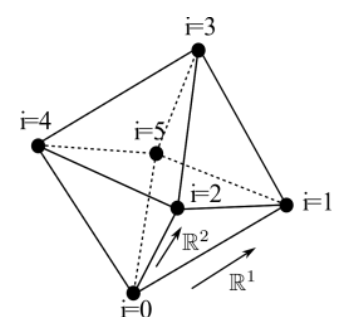

Fig. 9. Model the interconnected switches $i$ for 3-orthoplex.

As per the links among switches to obtain a quasi full mesh topology, some $2 N \cdot(N-1)$ links are needed, in a way that the nomenclature of the uplink ports of each switch will go from $M$ to $M+2 N-1$, except for port $M+i$ in each switch $i$, which would connect to itself, and also for port $M+((i+N) \bmod 2 N)$, which would go to the opposite node from $i$. This all may be appreciated in Fig. 10.

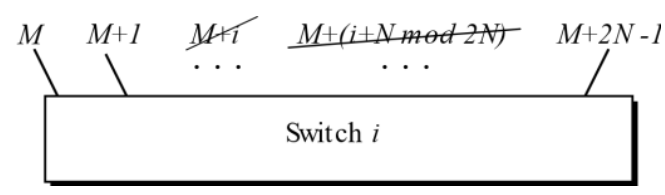

Fig. 10. Model for a generic switch $i$ for 3 -orthoplex with its uplink ports.

Eventually, taking all those schematic diagrams in mind, it is possible to express the user data traffic flows by means of pseudocode, as shown in Algorithm 2, which explains how a generic switch $i$ forwards incoming messages on towards destination host $b$.

Basically, it replicates the sequence of events exposed for the full mesh case scenario, but the difference goes when switch $i$ is the source switch $\operatorname{int}(a / M)$, where the packet gets forwarded on through all its uplink ports (thus getting redundancy), and those are not the same in quasi full mesh.

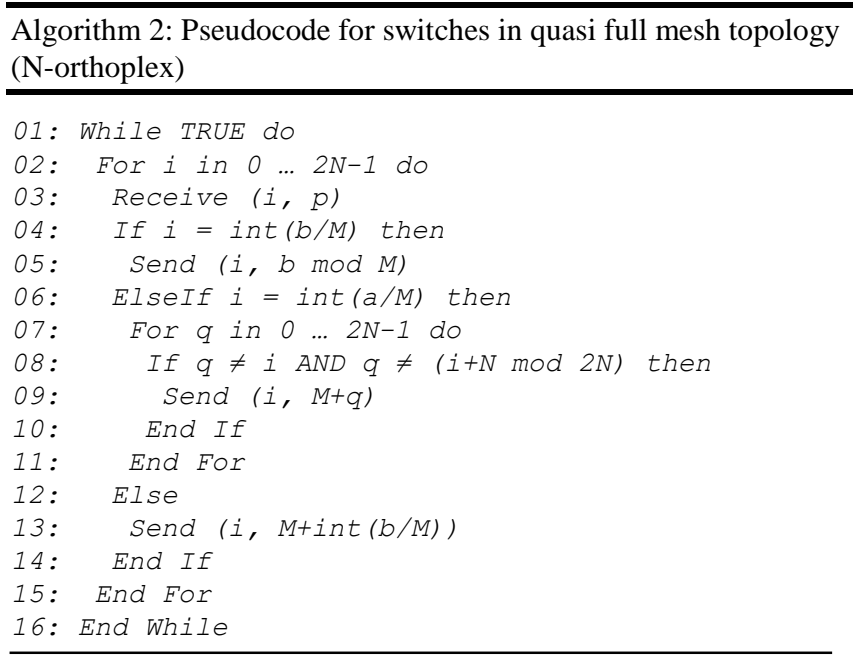

\section{VIII. $N$-HYPERCUBE: PARTIAL MESH}

Regarding the $N$-hypercube shape, its most interesting feature is that every node has just $N$ neighbors, each one being away in just one dimension, thus, having the same coordinates as such a node but not in just that specific dimension, regardless the dimension $N$, as seen in Fig. 11. 


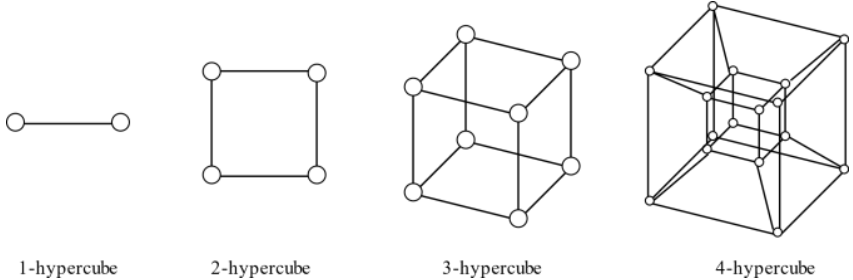

Fig. 11. $N$-hypercube for some values of dimension $N$.

This topology makes for a partial mesh topology, where a node $i$ has $N$ nodes at 1-hop away, and the rest of them are more than one hop away, accounting for several redundant paths, depending on the hop count from source node and destination node [10].

As per the number of $k$-facets contained in an $N$-hypercube of a given dimension $N$, it may be seen by expression (19).

$$
2^{N-k}\left(\begin{array}{l}
N \\
k
\end{array}\right)=2^{N-k} \cdot \frac{N !}{k ! \cdot(N-k) !}
$$

Applying the same reasoning as in the previous section, Table VII exhibits the main features for dimension $N$.

TABLE VII. AMOUNT OF K-FACETS PRESENT IN AN N-HYPERCUBE

\begin{tabular}{|c|c|c|c|c|c|c|}
\hline & $\begin{array}{c}(\varnothing) \\
k=-1\end{array}$ & $\begin{array}{c}\text { Nodes } \\
k=0\end{array}$ & $\begin{array}{c}\text { Edges } \\
\boldsymbol{k}=\boldsymbol{1}\end{array}$ & $\begin{array}{c}\text { Faces } \\
\boldsymbol{k}=\mathbf{2}\end{array}$ & $\begin{array}{c}\text { Cells } \\
\boldsymbol{k}=\mathbf{3}\end{array}$ & $\begin{array}{c}\text { 4-polyt. } \\
\boldsymbol{k}=\mathbf{4}\end{array}$ \\
\hline $\mathrm{N}$ & $2^{N+1}\left(\begin{array}{l}N \\
0\end{array}\right.$ & $2^{N}\left(\begin{array}{l}N \\
1\end{array}\right)$ & $2^{N-1}\left(\begin{array}{l}N \\
2\end{array}\right.$ & $2^{N-2}\left(\begin{array}{l}N \\
3\end{array}\right)$ & $2^{N-3}\left(\begin{array}{l}N \\
4\end{array}\right)$ & $2^{N-4}\left(\begin{array}{l}N \\
5\end{array}\right)$ \\
\hline-1 & 1 & N/A & N/A & N/A & N/A & N/A \\
\hline 0 & 1 & 1 & N/A & N/A & N/A & N/A \\
\hline 1 & 1 & 2 & 1 & N/A & N/A & N/A \\
\hline 2 & 1 & 4 & 4 & 1 & N/A & N/A \\
\hline 3 & 1 & 8 & 12 & 6 & 1 & N/A \\
\hline 4 & 1 & 16 & 32 & 24 & 8 & 1 \\
\hline
\end{tabular}

It is to be mentioned that the amount of $k$-facets in the $N$ hypercube are just those in the $N$-orthoplex, but flipped around, as both are dual of the other [11].

Focusing on the key features when working with Data Centre designs, such as the number of nodes and the number of edges, the proper expressions are shown in (20) and (21), respectively, which are equivalent to those cited in Table VII.

$$
\begin{gathered}
n^{\mathrm{o}} \text { nodes }=2^{N} \\
n^{\mathrm{o}} \text { edges }=N \cdot 2^{N-1}
\end{gathered}
$$

As per the hosts hanging on each switch $i$, the exposition stated in the previous section applies, depicted in Fig. 5, taking into consideration that the number of hosts per switch is $M$, which in turn, is supposed to be a power of 2 , such that $M=2^{L}$. This way, $2^{L}$ allows for easier arithmetic operations with $2^{N}$, which is the total amount of switches, making the overall number of hosts $2^{L+N}$, going from $h=0$ up to $h=2^{L+N}-1$.

With respect to the nomenclature of the switches $i$, being located at each vertex of the $N$-hypercube, one of them is identified with 0 , whereas the rest of the nodes are named by the expression $i=i_{0}+2^{j-1}$, where $\mathrm{i}_{0}$ may be a given node and $i$ accounts for all its $N$ neighboring nodes, taking $j$ in an incremental manner, starting from 1 and going all the way to $N$, thus standing for the real dimension where each neighbor is located respect to $\mathrm{i}_{0}$, as shown in Fig. 12 .

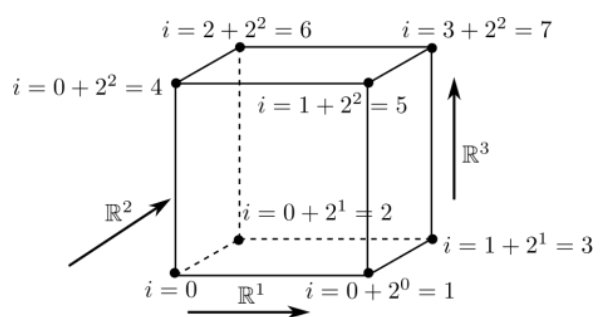

Fig. 12. Model the interconnected switches $i$ for 3-hypercube.

Looking at links among switches so as to get the plain $\mathrm{N}$ hypercube topology, there are $\mathrm{N} \cdot 2^{\mathrm{N}-1}$ links required overall, such that the nomenclature of the uplink ports only starts with port $M$ and ends up in port $M+2^{N}-1$ in each switch $i$, even though only $N$ ports are available, one for each neighbor, whilst the rest of ports are not being used, as seen in Fig. 13. Regarding the $\mathrm{N}$ uplink ports being used, those account for the expression port $M+\left(i+(-1)^{i n t\left(i / 2^{j-1}\right) \bmod 2} \cdot 2^{j-1}\right)$, as $j$ goes from 1 all the way to $N$, where $j-1$ may be swapped for $x$.

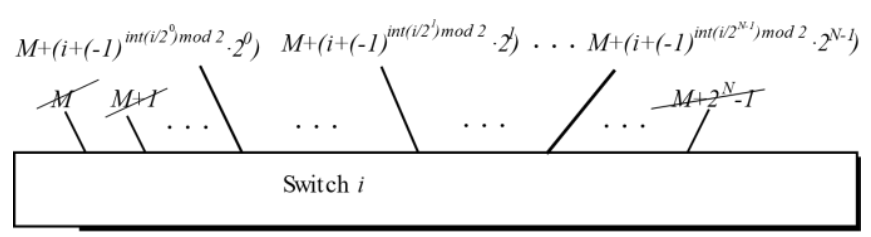

Fig. 13. Model for a generic switch i for N-hypercube with its uplink ports.

Eventually, taking all those schematic diagrams in mind, it is possible to express the user data traffic flows by means of pseudocode, as shown in Algorithm 3, which basically models how a generic switch $i$ deals with incoming messages to be forwarded on towards target host $b$.

Basically, it replicates the initial sequence of events exhibited for the full mesh case scenario, but the difference starts off if switch $i$ is not the destination switch $\operatorname{int}(b / M)$, and if this is the case, then the values of each bit positions $x$, going from bit 0 to bit $N-1$, of both switch identifiers in binary format are compared, where the former is given by the expression $\operatorname{int}\left(i / 2^{x}\right) \bmod 2$, whilst the latter is undertaken by expression $\operatorname{int}\left(\operatorname{int}(b / M) / 2^{x}\right) \bmod 2$. In this sense, for all mismatching bits $x$ being found out in that bit by bit comparison, then the packet is sent over the port $M+\left(i+(-1)^{\operatorname{int}\left(i / 2^{x}\right) \bmod 2} \cdot 2^{x}\right)$.

Algorithm 3: Pseudocode for switches in partial mesh topology (N-hypercube)

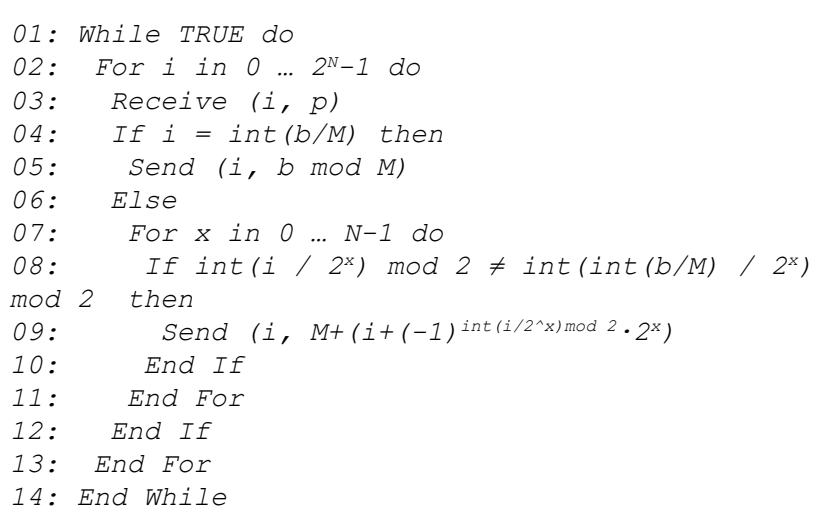


There is not a unique type of partial mesh topology as it allows for a great deal of variations, as long as all nodes are interconnected and some redundant paths among them are available. In this context, the $\mathrm{N}$-hypercube may be tuned up by adding up a brand-new link between any pair of opposite nodes, thus converting it into a folded $N$-hypercube topology, as it is depicted in Fig. 14. Opposite nodes may be calculated either by applying the 1's complement of a given switch $i$ in binary form, or by using the expression $2^{N}-i-1$ in decimal form. This way, the longest distance among nodes shrinks to $[N / 2]$.
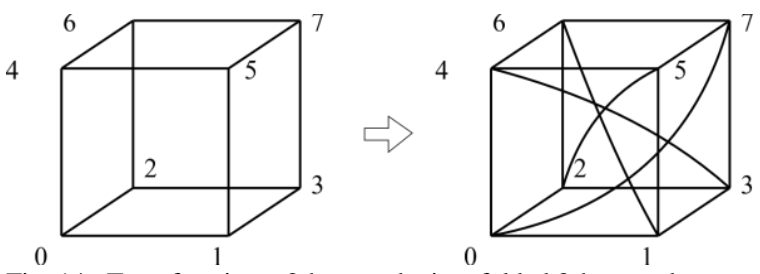

Fig. 14. Transforming a 3-hypercube in a folded 3-hypercube.

The downlink ports are the same as above, and so are the uplinks ports, but including a new port heading for each opposite node, being identified by $M+2^{N}$, as shown in Fig. 15 .

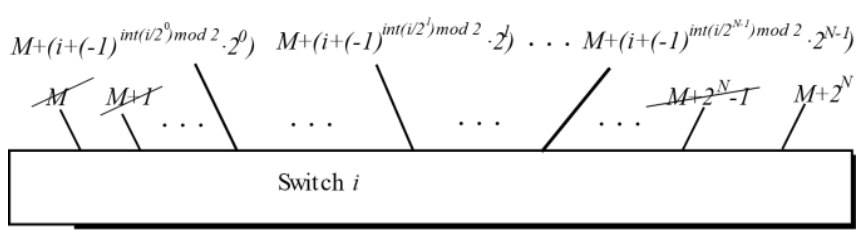

Fig. 15. Model for a switch $i$ for folded $N$-hypercube with its uplink ports.

Regarding the way, it deals with user data traffic, it is exhibited in Algorithm 4 by means of pseudo code. It works in a similar way as the plain $N$-hypercube, but if switch $i$ is not the destination switch, then variable distance calculates the number of mismatching bits between both switches. This value is compared to that of $N+1$-distance, and if the former is smaller, then the packet is forwarded on through the ports linked with the mismatching bits, or if the former it is greater, then it is sent over the ports assigned to matching bits, or else, if both are equal, then it is sent towards all those ports [12].

Algorithm 4: Pseudocode for switches in partial mesh topology (folded N-hypercube)

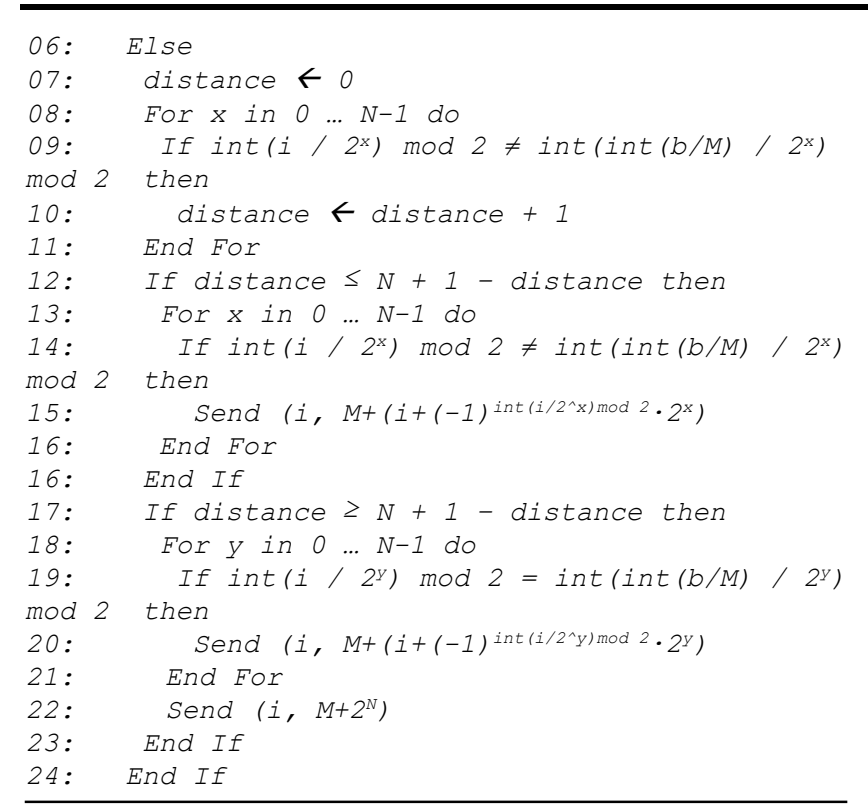

\section{CONCLUSIONS}

In this paper, multidimensional $N$-polytopes in a Euclidean space of dimension $N$ have been studied. To begin with, the Schläfli notation has been introduced in order to help classify the different types of regular $N$-polytopes in a recursive way, as long as their different underlying structures.

Moreover, the different regular $N$-polytopes for lower dimensions have been cited, finding that there are only three possible instances if $N \geq 5$ for convex regular ones, whereas there is no one for start regular ones.

Focusing on those three types for higher dimensions, they are known as $N$-simplex, $N$-orthoplex and $N$-hypercube. Each of those designs have been associated with a Data Centre topology, as their relevant features may correspond to those of full mesh, quasi full mesh and partial mesh, respectively.

For each of those topologies, a summary of its features has been quoted, and in turn, the nomenclature for identifying the different switches within the topology has been cited, along with its downlink and uplink ports. Besides, a common model to identify end hosts for all topologies has also been named.

At that point, an algorithm describing how a given switch deals with user data traffic within each of those topologies have been presented. Specifically, an arithmetic approach has been applied in a generic switch so as to guide incoming traffic coming in from any port towards destination by forwarding that traffic out through the appropriate port, or more than one port if equal path load balancing is available, according to some relevant arithmetic expressions.

Therefore, three different algorithms have been presented, based on an arithmetic approach, to forward incoming traffic into a particular switch through its proper outgoing ports making for the shortest available paths to the destination host.

\section{REFERENCES}

[1] P. Habibi et al, "Fog Computing: A Comprehensive Architectural Survey," IEEE Access, vol.8, pp. 69105-69133, 2020. DOI: 10.1109/ACCESS.2020.2983253

[2] E. Oughton et al, "Revisiting Wireless Internet Connectivity: $5 \mathrm{G}$ vs WiFi 6," Telecommunications Policy, vol.45, no.5, pp. 1-15, 2021. DOI: 10.1016/j.telpol.2021.102127

[3] M. Waqas et al, "Mobility-Aware Fog Computing in Dynamic Environments: Understandings and Implementation," IEEE Access, vol. 7, pp. 38867-38879, 2018. DOI: 10.1109/ACCESS.2018.2883662

[4] C.S.R. Prabhu, "Overview - Fog Computing and Internet-of-Things (IoT)," EAI Endorsed Transactions on Cloud Systems, vol.3, no.10, pp. 1-24, 2017. DOI: 10.4108/eai.20-12-2017.154378

[5] O. Osanaiye, S. Chen, Z. Yan, R. Lu, K.R. Choo and M. Dlodlo, "From Cloud to Fog Computing: A Review and a Conceptual Live VM Migration Framework," IEEE Access, vol.5, pp. 8284-8300, 2017. DOI: 10.1109/ACCESS.2017.2692960

[6] A. Neeman, Algebraic and Analytic Geometry. Cambridge University Press, 2007, Canberra, Australia. DOI: 10.1017/CBO9780511800443

[7] D.M.Y. Sommerville, An introduction to the Geometry of $N$ Dimensions. Dover Publications Inc., 2020, New York, U.S.A.

[8] R. Souam, "The Schläfli formula for polyhedra and piecewise smooth hypersurfaces," Differential Geometry and its Applications, vol.20, no.1, pp. 31-45, 2004.

[9] A. Tanenbaum, Computer Networks. Prentice Hall Internat, 2002, New Jersey, U.S.A.

[10] B. Forouzan et al., Data Communications and Networking. McGraw Hill, 2000, New York, U.S.A.

[11] P.J. Roig, S. Alcaraz, K. Gilly and C. Juiz, "Modelling a Plain NHypercube Topology for migration in Fog Computing," Lecture Notes in Electrical Engineering, accepted by not published yet.

[12] P.J. Roig, S. Alcaraz, K. Gilly, C. Juiz, "Modelling a Folded NHypercube Topology for migration in Fog Computing," Lecture Notes in Electrical Engineering, accepted by not published yet. 